Cahiers $d u$ MONDE RUSSE

\section{Cahiers du monde russe}

Russie - Empire russe - Union soviétique et États indépendants

$51 / 4 \mid 2010$

Sciences humaines et sociales en Russie à l'Âge d'argent

\title{
Michael John, Die Anfänge des sozialistischen Realismus in der sowjetischen Musik der 20er und 30er Jahre
}

\section{Gábor T. Rittersporn}

\section{(2) OpenEdition}

\section{Journals}

Édition électronique

URL : https://journals.openedition.org/monderusse/7387

DOI : $10.4000 /$ monderusse. 7387

ISSN : $1777-5388$

Éditeur

Éditions de l'EHESS

Édition imprimée

Date de publication : 25 novembre 2010

Pagination : 709-713

ISBN : 978-2-7132-2316-7

ISSN : $1252-6576$

Référence électronique

Gábor T. Rittersporn, « Michael John, Die Anfänge des sozialistischen Realismus in der sowjetischen Musik der 20er und 30er Jahre », Cahiers du monde russe [En ligne], 51/4 | 2010, mis en ligne le 09 décembre 2011, consulté le 03 septembre 2022. URL : http://journals.openedition.org/monderusse/ 7387 ; DOI : https://doi.org/10.4000/monderusse.7387

Ce document a été généré automatiquement le 3 septembre 2022

Tous droits réservés 


\title{
Michael John, Die Anfänge des sozialistischen Realismus in der sowjetischen Musik der 20er und 30er Jahre
}

\author{
Gábor T. Rittersporn
}

\section{RÉFÉRENCE}

Michael JонN, Die Anfänge des sozialistischen Realismus in der sowjetischen Musik der 20er und 30er Jahre. Historische Hintergründe, ästetische Diskurse und musikalische Genres. Bochum - Freiburg : Projekt Verlag, 2009, 660 p.

1 Dans son Guignol antiformaliste, Šostakovič a récapitulé succinctement ce que les autorités soviétiques attendaient de la musique réaliste socialiste: beauté, élégance, mélodie, polyphonie, noblesse et formes classiques. Le compositeur cite les diatribes de quelques critiques qui ne savaient manifestement pas le moins du monde comment distinguer la musique authentiquement soviétique de celle qu'avaient pratiquée tous les artistes pendant des siècles. Šostakovič fait également référence aux élucubrations d'Andrej Ždanov sur la lezginka qui devait être "expressive", mais sa propre énumération est beaucoup plus riche que la liste de l'illustre pourfendeur d'un formalisme soi-disant antipopulaire. Car le gardien de la pureté de l'art musical avait toutes les peines du monde à accoucher de termes tels que " simplicité », «intelligibilité de la forme » ou encore «sonorité naturelle de la parole». Allez donc savoir ce qu'on peut entendre par réalisme socialiste en musique!

2 Dans les années 1970, l'Encyclopédie musicale soviétique en dit encore moins : le court article sur la question apprend au lecteur qu'il s'agit de la « forme suprême du réalisme », qui apparaît dans des «formes populaires originales ». Mais, pour ne pas laisser les curieux sur leur faim, les rédacteurs prennent le soin de mentionner, dans l'article quelque peu 
tortueux sur le réalisme, que certaines œuvres de Sergej Prokof'ev, Ivan Dzeržinskij et Tihon Hrennikov sont imprégnées d'humanisme socialiste et de réalisme. Les auteurs n'oublient pas Šostakovič et sa Lady Macbeth du district de Mcensk qui avait provoqué l'ire de Stalin en 1936, mais était devenue digne du titre honorifique de « réaliste » et même vaguement de celui de «socialiste » quarante ans plus tard. Notons que Dzeržinskij et Hrennikov sont ridiculisés dans le Guignol, non sans raison. Notons également qu'en 1933, Šostakovič avait publié un article où il regrettait que la critique musicale tarde à définir le réalisme socialiste, qui avait pourtant déjà agité des esprits aussi agiles que Lunačarskij. Notons enfin que les auteurs de l'article de la dite encyclopédie mentionnent en passant qu'au milieu des années 1950, l'esthéticien Aleksandr Burov s'était risqué à nier que le concept de réalisme s'applique à la musique.

3 Cela valait peut-être la peine de s'attarder sur ces détails pour rappeler que même des sommités aussi éminentes en musicologie que Ždanov peinaient à définir ce que les autorités du pays des Soviets exigeaient des compositeurs. Ajoutons tout de suite qu'ils peinaient tout autant à dire ce dont ils ne voulaient pas, car le concept élastique de formalisme qu'ils dénonçaient n'était pas moins flou que celui de réalisme socialiste. Il n'est pas étonnant que les musiciens se soient parfois égarés du droit chemin, tout en étant convaincus de suivre les préceptes de la Zukunftsmusik bolchevique. Car il faut vraiment y mettre du sien pour repérer ce qui relève du réalisme socialiste dans le paysage accidenté de la musique du $\mathrm{xx}^{\mathrm{e}}$ siècle.

4 Même un connaisseur aussi fin de la musique russe et soviétique que Michael John prend des risques dès lors qu'il s'essaie à l'aventure. Cet ouvrage sur les débuts du réalisme socialiste dans la musique soviétique témoigne d'une érudition extraordinaire. Ainsi, les chapitres qu'il consacre aux motifs et aux genres populaires en musicologie, dans les chants d'église et dans l'évolution de la pédagogie musicale en Russie au xix ${ }^{\mathrm{e}}$ siècle, ouvrent de nouveaux horizons pour étudier les tendances qui ont influencé nombre d'idées postrévolutionnaires. L'auteur dévoile un visage peu connu du public étranger d'Aleksandr Kastal'skij, compositeur novateur de chœurs d'église, musicien ouvert sur le panthéisme populaire et pionnier dès les années 1880 du mouvement qui voulait " apporter la musique au peuple ». Rares sont les historiens sachant qu'après 1917, Kastal 'skij a rejoint le Proletkult, contribué à révolutionner l'enseignement musical et élaboré une théorie fort originale de la polyphonie populaire russe. Par ailleurs, John place dans une nouvelle perspective la musicologie soviétique de l'entre-deux-guerres. Son analyse de divers genres musicaux des années 1920 et 1930 est d'une subtilité impressionnante.

Mais sa quête du Saint-Graal du réalisme socialiste risque fort de ne déboucher nulle part. C'est que les structures, systèmes tonaux, harmonies ou formules rythmiques qui ressortent des analyses de John n'ont rien à voir avec quelque socialisme que ce soit et, surtout, ne caractérisent pas que les genres qu'il définit comme réalistes socialistes. Par exemple, John a parfaitement raison de souligner que le chant de masse soviétique était censé pénétrer le quotidien et fonder une mémoire collective, ce qui supposait des formes simples, claires et faciles à mémoriser. Mais on peut se demander si les chants révolutionnaires et ceux des mouvements radicaux et ouvriers composés à l'étranger ne répondaient pas aux mêmes objectifs et n'étaient pas de la même facture, au moins depuis les guerres paysannes des pays germaniques au Xvi siècle et surtout depuis la Révolution française. L'auteur évoque l'adaptation ou la simplification des chants du mouvement ouvrier international en Russie et en Union soviétique, mais sans aller plus loin. 
Il en va de même du sous-genre des chansons pseudo-folkloriques qui tantôt glorifiaient le kolkhoze, Stalin ou les deux ensemble, tantôt évoquaient de tendres sentiments en notant tout au plus que leur objet était stakhanoviste, conducteur de machines agricoles ou guerrier de l'Armée rouge, mais sans célébrer l'agriculture collectivisée et son plus éminent champion. Un pseudo-folklore florissait en Europe de l'Est depuis le début du $\mathrm{XIX}^{\mathrm{e}}$ siècle. Les réminiscences folkloriques des chansons de Vladimir Zaharov ou de Matvej Blanter n'ont rien à envier au genre du magyar nóta hongrois et à son alla zingarese qui rappelle quelque chose de celle de Brahms, sans égaler, loin de là, la maîtrise des formes et de l'orchestration ainsi que l'ironie géniale de ce compositeur.

7 Ce n'est malheureusement qu'en note de bas de page que John indique que les chants symphoniques soviétiques devaient beaucoup à Beethoven. Il relève fort judicieusement l'appauvrissement de la forme de la sonate dans la symphonie soviétique, dès que le compositeur voulait susciter l'émotion de l'auditeur au lieu de lui faire suivre le développement des motifs in extenso. Mais que dire d'autre de la musique symphonique et des sonates, disons, de Miklós Rózsa, grand prince des compositeurs d'Hollywood, ainsi que des œuvres de tant d'autres artistes étrangers qui penchaient plutôt vers des effets faciles pour éviter de mettre trop à l'épreuve la capacité de concentration de leur public?

8 John ne parle pas de l'opéra soviétique, ce qui aurait pourtant permis de réfléchir à un parallèle plus que probable entre le souci des compositeurs et de la politique culturelle en URSS d'en faire un genre phare, et l'impératif obsessionnel à l'est de l'Europe, au $\mathrm{XIX}^{\mathrm{e}}$ siècle, de créer un opéra national. Pourtant, le genre était tellement porteur que même Isaak Dunaevskij avait l'intention de l'utiliser. John ne tente pas de récupérer la musique de ce compositeur pour le réalisme socialiste. Et pour cause, car celle-ci devait autant au genre du chant de masse qu'au jazz, à la romance russe, à Odessa, à Hollywood ou à l'opérette viennoise. L'opérette soviétique échappe aussi au champ de vision de l'auteur, peut-être parce qu'elle avait beaucoup à voir avec un cocktail stylistique à la Dunaevskij et avec le pseudo-folklore. Mais elle aurait mérité un petit détour, ne seraitce que pour se demander pourquoi Imre Kálmán et Ferenc Lehár ont été aussi populaires au pays du réalisme socialiste que sous la monarchie austro-hongroise.

Il se peut que John ne soit pas aussi sûr de son fait qu'il n'y parait. En tout cas, il insiste beaucoup sur l'importance des paroles des chants en expliquant qu'elles devaient interpeller différents groupes sociaux. Il s'agissait d'engager différents auditoires dans le chœur immense, à plusieurs voix, des peuples de l'URSS, qui chantaient ici les mérites des héros du travail, là ceux des kolkhoziens ou des jeunes pionniers, et partout le génie du chef suprême et les miracles qu'on associait à son nom. John constate que peu importait le style musical - qui allait de l'imitation des chansons populaires jusqu'à celle du swing, précisément par Dunaevskij-, car c'est le texte qui contient le véritable message que les moyens musicaux sont censés aider à mémoriser et à transformer en expérience quotidienne.

10 Mais cela revient à dire que le réalisme socialiste des œuvres ne relevait pas vraiment d'un langage musical spécifique mais des spécificités de la littérature. Tout le problème est là, car la théorie du réalisme socialiste était échafaudée pour des genres littéraires et dramatiques qui intégraient les scénarios de films mais certainement pas l'architecture ou l'ikebana, ni même les moyens stylistiques de la cinématographie. Or ces derniers ne sont pas plus capables d'exprimer ce que les théoriciens du réalisme socialiste taxaient de développement révolutionnaire de la réalité que ceux de la musique. Ce n'est pas pour 
rien que les autorités de l'industrie cinématographique s'intéressaient avant tout au texte des dialogues et à ce que suggérait l'intrigue, à l'instar du censeur suprême, Stalin, qui intervenait souvent pour critiquer les scénarios mais ignorait magistralement les plans, l'éclairage, le montage ou le son qui fondent le film.

C'est pourquoi John se lance dans une entreprise hasardeuse quand il tente de dégager le message idéologique caché (et évident) dans les constructions cantiques et symphoniques des films de Grigorij Aleksandrov et de Dziga Vertov. Il n'y a aucun problème en ce qui concerne le message. En effet, celui-ci est plus ou moins habilement masqué dans les comédies musicales d'Aleksandrov et fièrement exhibé chez Vertov. Mais le malheur veut que chez le premier, ce sont l'action, les dialogues et les paroles des chansons qui le font (ou non) parvenir jusqu'au spectateur, alors que chez le second, le discours idéologique est exposé par les éléments symboliques et narratifs de l'image, par les intertitres qui les annoncent ou les interprètent et par les véritables discours que les acteurs prononcent. John ne peut pas contourner ces moyens éminemment littéraires et se sent acculé à en parler beaucoup, même si son excellente analyse des structures cinématographiques fait ressortir la musicalité des styles. Mais pourquoi évoque-t-il l'inspiration hollywoodienne des comédies et toute la panoplie stylistique de l'avantgarde cinématographique internationale ? Fort rares étaient les films réalistes socialistes au-delà des frontières jalousement gardées de l'URSS, malgré le recours fréquent des cinéastes du monde entier aux moyens que Vertov a mis en œuvre.

L'analyse des films contient ici et là quelques remarques sur la musique sans s'y attarder, même quand il s'agit d'opérettes filmées. Pourtant, il aurait certainement été judicieux que John étudie les fonctions des marches tonifiantes et des colonnes de travailleurs, soldats et jeunes pionniers dans l'Enthousiasme (Symphonie du Donbass), et qu'il s'interroge sur les chants qui mobilisaient les masses, au début des années 1930, en Europe centrale, occidentale, du Sud et de l'Est, y compris à partir des écrans de cinéma. De même, la belle analyse de la forme du film Quatre chants sur Lenin ne fait aucune référence à la musique qui illustre les images. Or celle-ci donne du fil à retordre dès lors qu'on veut parler de réalisme socialiste. Des extraits du futur opéra Terres défrichées d'Ivan Dzeržinskij y cohabitent paisiblement avec les mélodies du Chant funèbre et du Chant de jubilation d'Aleksandr Veprik, de même que des morceaux de la cantate (dans le style « chants de masse ») de Marian Koval' sur Pugačev (alors en gestation) côtoient la marche funèbre de Siegfried du Crépuscule des dieux qu'on entend de la première note jusqu'à la dernière dans les plans d'un film d'actualités sur l'enterrement de Lenin. Veprik, compositeur fascinant mais peu joué en Russie comme à l'étranger, Dzeržinskij et Koval' sont des représentants de styles fort différents et n'ont pas le même talent, loin s'en faut, mais tous trois ainsi que leur public étaient convaincus qu'ils pratiquaient une certaine musique soviétique. Et que faire de Siegfried ? Certes, en 1924, sa marche funèbre fut exécutée à la réunion qui suivit les obsèques de Lenin, au Bolchoï. Dix ans plus tard, Vertov n'aurait-il pas eu de musique authentiquement soviétique à sa disposition pour accompagner dignement les funérailles du fondateur de l'État soviétique dans un film relevant du réalisme socialiste ? Ou pour lui, le Ring de Wagner aurait-il été aisément assimilable au réalisme socialiste? Autant de questions sur lesquelles John méditera peut-être un jour.

13 Finalement, il semblerait que confronter la musique soviétique avec les courants stylistiques d'autres sphères culturelles soit plus fructueux que de la maintenir clouée sur le lit de Procuste du réalisme socialiste. Même si nombre de compositeurs soviétiques se défendaient de suivre d'autres modèles, ils le faisaient, de toute évidence, 
et Šostakovič n'était certainement pas le seul à être conscient de ce qui le liait à une tradition internationale. Même son Guignol antiformaliste n'était pas sans précédent. Déjà, en 1925, Schönberg se raillait des modes musicales de l'époque dans ses Trois satires pour chœur mixte et égratignait Stravinskij, sans pour autant l'accuser de succomber aux charmes d'une quelconque Zukunftsmusik soviétique. 